\title{
Empfehlung zur Behandlung respiratorischer Komplikationen bei einer Viruspandemie
}

\author{
D. Köhler \\ O. Karg \\ J. Lorenz \\ R. Mutters \\ T. Schaberg \\ B. Schönhofer \\ T. Welte
}

\author{
Recommendations for the Treatment of Respiratory Complications \\ in Case of a Viruspandemic
}

\section{Zusammenfassung}

Bei einer Pandemie mit Viren, für die es keinen Impfstoff gibt, ist im ungünstigsten Falle mit einem Massenanfall von schwer Erkrankten von 250 - 300 Neuaufnahmen pro Woche pro Krankenhaus zu rechnen. Im Vordergrund stehen dabei respiratorische Komplikationen, insbesondere Pneumonien, oft mit bakterieller Superinfektion. Nicht selten müssen diese Patienten passager beatmet werden. Die jetzige Infrastruktur erlaubt aber nicht, einen solchen Massenanfall adäquat zu behandeln. Basierend auf den großen Erfolgen, welche die nichtinvasive Beatmung bei akuter respiratorischer Insuffizienz in den letzten Jahren erzielen konnte, ist diese Empfehlung der Deutschen Gesellschaft für Pneumologie entstanden. Es wird Wert darauf gelegt, mit vergleichsweise einfachen Mitteln größere Patientenkollektive effektiv zu behandeln. Dabei wird empfohlen, ein separates Gebäude bereitzustellen, damit eine Quarantäne möglich ist. An diagnostischen Mitteln sollte der Zugang zu einfachen Röntgenaufnahmen möglich sein. Für die Patientenüberwachung reicht in den meisten Fällen eine Pulsoxymetrie; mitunter ist auch ein EKG-Monitoring erforderlich. Für die Therapie der respiratorischen Insuffizienz sollen eine entsprechende Anzahl von Beatmungsgeräten mit Masken, Schlauchsystemen und Filtern vorgehalten werden. Bei der medikamentösen Behandlung stehen Antibiotika zur Behandlung der Superinfektionen im Vordergrund. Analgetika, Sedativa und Flüssigkeitssubstitution müssen ebenfalls gelagert werden. Alle Patienten sollten die Möglichkeit der Sauerstoffapplikation haben. Die Empfehlung gibt detaillierte Hinweise zur praktischen Durchführung der Hygiene, Diagnostik und Therapie bei einem Massenanfall von Viruserkrankungen im Krankenhaus.

\section{Abstract}

In case of a viral pandemic without availability of effective vaccination, one can expect to be faced with additional 250 to 300 new admissions per hospital per week given the worst case scenario. Major complications are expected to occur in the respiratory system with the focus on viral pneumonia often complicated by bacterial superinfection. Frequently these patients will require artificial ventilation. The present infrastructure will not be capable of dealing sufficiently with such high numbers of casualties. These recommendations of the German Society for Pneumonology are based on the successful application of non-invasive ventilation for acute respiratory failure in recent years. It is of importance to achieve effective treatment by the use of relative simple means. The recommendation proposes to use a separate building in order to realize quarantine. In terms of diagnostic tools, a simple x-ray apparatus should be available. To monitor patients pulsoxymetry and ECG devices should be sufficient in most cases. For the treatment of acute respiratory insufficiency a sufficient number of ventilators, masks, tubing systems and filters should be kept in stock. In terms of medical treatment antibiotics to treat superinfections are of major importance. Analgesics, sedatives and intravenous fluids will also be needed. Oxygen should be available for every single patient. The recommendation gives detailed advise for the enforcement of hygiene control, diagnostic as well as therapeutic steps for in hospital treatment of high numbers of casualties of a viral pandemic. 
Mutationen des Virusgenoms können zu Varianten führen, gegen die keine protektive Immunantwort des Wirtes möglich ist. Dies kommt bei Influenzaviren, aber zum Beispiel auch bei Coronaviren (SARS-CoV) vor. Das gleichzeitige Vorkommen der aviären Influenza und der humanen Influenza A in Südostasien erhöht die Wahrscheinlichkeit einer genetischen Rekombination. Dies birgt das Risiko einer Pandemie in sich. Wenn die Viren nicht oder nur wenig auf Virostatika ansprechen oder kein Impfschutz möglich ist, kann die Situation zu einer internationalen Katastrophe führen. Näheres hierzu findet sich auf den Seiten des Robert-Koch-Institutes. Insbesondere sind hier die wesentlichen Aspekte im nationalen Influenza-Pandemieplan (unter www-rki.de) zusammengestellt.

Je nach Infektiösität und möglicher Ausbreitungsgeschwindigkeit sind verschiedene Szenarien bei einer Pandemie denkbar, wobei, bezogen auf Deutschland, im ungünstigen Falle mit bis zu 250-300 Neuaufnahmen pro Woche pro Krankenhaus zu rechnen ist. Dieses kann mit der üblichen Krankenhausinfrastruktur derzeit in keiner Weise bewältigt werden. Meist stehen bei den Grippe-Erkrankten respiratorische Komplikationen im Sinn einer Viruspneumonie bzw. einer Pneumonie durch Superinfektion im Vordergrund, da sich der Virus i.d.R. als Aerosol ausbreitet $[10,15]$. Beobachtet werden aber auch schwere Laryngotracheitiden und Exazerbationen von vorbestehenden Bronchialerkrankungen wie vor allem COPD und Asthma. Manche Viren führen sofort zu systemischer Vaskulitis mit Multiorganversagen [14].

Viele dieser Patienten können heute mit der erst in den letzten Jahren von der Pneumologie in der Breite eingeführten nicht-invasiven Beatmung über die kritische Phase gebracht werden [2-6,11]. Durch diese Möglichkeit ist jetzt eine wesentlich einfachere Infrastruktur zur Behandlung der schwerer Erkrankten ohne Intensivstation erforderlich. Hinzu kommt, dass darunter die Ventilator-assoziierte Pneumonie wesentlich seltener vorkommt $[7,11]$. Die Deutsche Gesellschaft für Pneumologie und Beatmungsmedizin möchte mit diesen Empfehlungen Hilfestellung für die regionalen Katastrophenpläne geben, die für einen solchen Massenanfall von Erkrankten derzeit erstellt werden.

\section{Lokalisation}

Die Unterbringung der schwerer erkrankten Patienten mit Verdacht auf lebensbedrohliche Komplikationen (i.d.R. Pneumonie) sollte möglichst in einem separaten Gebäude erfolgen, damit eine Quarantäne möglich ist. Vorteilhaft sind eigene Gebäude im Klinikbereich (z. B. Psychiatrie) oder eine Reha-Klinik, die rasch komplett geleert werden kann. Hotels oder ähnliche Einrichtungen sind wegen fehlender Sauerstoffversorgung und sanitärer Möglichkeiten deutlich weniger geeignet. Die Gebäude benötigen eine Schleuse für alle, die sie betreten oder verlassen. Bauliche und hygienische Voraussetzungen sind in dem Pandemieplan des RKI veröffentlicht.

\section{Personal}

Im Pandemiefall wird über das in Krankenhäusern vorhandene noch zusätzliches Personal benötigt. Deswegen sollten vorher im regionalen Katastrophenplan entsprechende Pflegekräfte und Ärzte erfasst werden, die in der Region wohnen, derzeit aber nicht in Krankenhäusern oder gar nicht arbeiten. Auch an Hilfskräfte mit Erfahrung im medizinischen Bereich, wie frühere Zivildienstleistende, Medizinstudenten und Rotkreuzhelfer, sollte gedacht werden. Der Personalschlüssel muss von den regionalen Gegebenheiten abhängig gemacht werden.

\section{Diagmostik}

Die Patienten kommen neben dem schweren grippetypischen Krankheitsgefühl mit Muskelschmerzen meist mit akut einsetzender Luftnot und/oder Ödemen zur Aufnahme. Von großer Bedeutung ist die Möglichkeit der Durchführung von einfachen Röntgen-Thoraxaufnahmen. Alle Kliniken verfügen über mobile Röntgeneinheiten, wie sie z.B. auf Intensivstationen oder in der Endoskopie zum Einsatz kommen. Ebenfalls ist ein Durchleuchtungssystem praktisch immer verfügbar. Die Durchleuchtung hat den großen Vorteil, dass keine Röntgenkassetten erforderlich sind, die in der Regel außerhalb der Quarantäneeinheit entwickelt werden müssen. Digitale Systeme dürften in den Quarantäneeinrichtungen in absehbarer Zeit nicht verfügbar sein. Allerdings gibt es zunehmend mobile Einheiten, die Papierausdrucke der Röntgenbilder am Gerät ermöglichen.

Auf der anderen Seite haben Durchleuchtungssysteme den Nachteil einer deutlich reduzierten Qualität. Röntgenaufnahmen mit einem mobilen System sind auch ohne Rasterautomatik qualitativ besser als die Durchleuchtung. Hier stellt sich das Problem des Transportes der belichteten Kassette in die Klinik zur Entwicklung oder zum digitalen Auslesen. Es wird vorgeschlagen, einen entsprechenden Vorrat an Kassetten anzulegen, die in eine Folie eingeschweißt oder in einer entsprechenden verschließbaren Folientüte verpackt werden können. Aus diesen Tüten können die Kassetten dann ausgeschleust werden.

Nicht selten haben die Patienten mit Pneumonie Pleuraergüsse. Diese können mitunter so erheblich sein, dass sie für die respiratorische Insuffizienz verantwortlich sind. Deswegen sollte die Möglichkeit einer Ultraschalldiagnostik vor Ort existieren. Über Pleuradrainagen kann hier relativ rasch entlastet werden. Das Ultraschallgerät erlaubt darüber hinaus heute eine effiziente Diagnostik vieler anderer Organsysteme. Die Diagnose von Lungeninfiltraten mittels Ultraschall ist unter bestimmten Voraussetzungen auch möglich, dürfte jedoch im Notfall kaum praktikabel sein, da sie eine große Erfahrung erfordert. Zudem sind viele Infiltrate nicht sichtbar, wenn sie keine Verbindung zur Pleura haben.

Zur Erfassung von Rhythmusstörungen sollte ein EKG-Gerät verfügbar sein, außerdem eine Defibrillatoreinheit. Ein zentrales Element der Versorgung der respiratorisch Erkrankten ist die Pulsoxymetrie. Damit können viele wesentliche Informationen gewonnen werden, die zur Überwachung bzw. zur Beatmungstherapie erforderlich sind. Blutgase sind damit i.d. R. entbehrlich. 
Zur weiteren Basisausstattung gehören ausreichend Fieberthermometer und Blutdruckgeräte. Eine Labordiagnostik ist in begrenztem Umfang vor Ort möglich, wenn Notfalllaborsysteme zur Verfügung stehen. Wichtig ist, für entsprechenden Vorrat an Blutabnahmesystemen zu sorgen, die nach Desinfektion in geschlossenen Boxen für infektiöses Material ausgeschleust werden können.

Bronchoskope mit Batteriebetrieb vorzuhalten - wie sie zunehmend auf den Intensivstationen eingesetzt werden - wäre von großem Vorteil. Dazu muss allerdings in der Quarantänestation auch eine eigene Desinfektionseinheit vorhanden sein.

\section{Hygiene}

Die aktuellen Empfehlungen zu den Hygieneanforderungen finden sich als Merkblatt auf der Homepage des RKI. Die grundsätzlichen Anforderungen sollen hier kurz genannt werden:

Wegen der aerogenen Übertragung des Virus ist eine Unterbringung in Einzelzimmern anzuraten. Dies ist im Falle einer Pandemie allerdings oft nicht zu leisten, so dass eine Kohortenisolierung mit ihren höheren Anforderungen an die Sicherheit der versorgenden Mitarbeiter der Regelfall sein wird. Wenn möglich sollten die Zimmer oder Bereiche mit Schleusenfunktion ausgestattet sein. Sofern in den Räumen eine raumlufttechnische Anlage betrieben wird, ist diese abzustellen.

\section{Personalschutz}

Um die Aufnahme von Aerosolen zu verhindern, ist vom medizinischen Personal ein dicht sitzender Mund-Nasenschutz zu tragen. Dieser sollte nach Möglichkeit der Schutzstufe FFP3 entsprechen. Zu den sinnvollen und notwendigen Personalschutzmaßnahmen gehört zudem das Tragen von geschlossenen Schutzkitteln und Einweghandschuhen. Empfehlenswert sind hier virusdichte Nitritacetat-Handschuhe. Schutzkittel, MundNasenschutz, Einmalhandschuhe sind im Zimmer des Patienten, bzw. in der Schleuse anzulegen. Handschuhe und Mund-Nasenschutz werden in geschlossenen Behältnissen entsorgt, der Schutzkittel wird der sicheren Aufbereitung zugeführt. Die Händedesinfektion wird mit einem auf Viruswirksamkeit geprüften Desinfektionsmittel nach direktem Patientenkontakt, nach Kontakt mit erregerhaltigem Material oder kontaminierten Objekten sowie nach Ablegen der Handschuhe durchgeführt.

\section{Flächendesinfektion und Reinigung}

Um eine Anreicherung der Viruslast auf Flächen zu unterbinden, ist eine tägliche Scheuerwischdesinfektion der patientennahen Flächen mit geprüften und viruswirksamen Mitteln notwendig. Bei Bedarf sind diese Maßnahmen auf weitere kontaminationsgefährdete Flächen auszudehnen. Alle Medizinprodukte mit direktem Kontakt zum Patienten (z. B. EKG-Elektroden, Stethoskope, etc.) sind patientenbezogen zu verwenden und müssen nach Gebrauch desinfiziert werden. Bei Transport in einem geschlossenen Behälter ist eine zentrale Aufbereitung möglich. Thermische Desinfektionsverfahren sollten bevorzugt angewendet werden. Wäsche/Textilien können desinfizierend gewaschen werden. Für Betten und Matratzen werden wischdesinfizierbare Überzüge empfohlen.

\section{Transport}

Ist ein Transport von Patienten im Krankenhaus unvermeidbar, so ist der Zielbereich vorab zu informieren. Der Patient muss einen Mund-Nasen-Schutz tragen (bei partikelfiltrierenden Halbmasken selbstverständlich ohne Ventil), das Personal trägt die o.a. Schutzmittel. Unmittelbar nach den erfolgten Maßnahmen sind die Kontaktflächen und das Transportmittel vor erneuter Nutzung zu desinfizieren.

\section{Maßnahmen bei Tod}

Im Falle des Versterbens eines Patienten ist der Leichnam in die entsprechenden für HKLE (Hoch-Kontagiöse-LebensgefährlicheErkrankungen) und SARS vorgesehenen Transportsäcke zu verbringen, um somit ein Infektionsrisiko auszuschließen.

\section{Labor}

Ein oft übersehenes Problem sind Einrichtungen der mittelbaren Krankenversorgung, wie Labors der Klinischen Chemie, Mikrobiologie, Virologie. Es ist sicherzustellen, dass sämtliches Untersuchungsmaterial in geschlossenen Transportbehältnissen in die Labore transportiert wird. Diese dürfen außen nicht mit biologischem Material kontaminiert sein und müssen als infektiös gekennzeichnet werden.

\section{Therapie}

\section{Medikamente}

Wenn die Pandemie eine Region erreicht hat, ist in der Regel bekannt, um welchen Virus es sich handelt und ob Virustatika, insbesondere Neuraminidasehemmer, ansprechen. Eine Bevorratung mit den notwendigen Virustatika ist unbedingt anzuraten. Favorisiert wird Oseltamivir, da es breit wirksam ist und die Wirkung unabhängig vom meist stattfindenden Antigendrift bei neuen Epidemiestämmen sowie infolge der systemischen Gabe sicherer eintritt. Es ist darauf zu achten, dass das medizinische Personal entsprechend prophylaktisch mitbehandelt wird. Nur so ist eine Aufrechterhaltung des Klinikbetriebes sicher zu stellen.

Häufig haben die Patienten sekundäre bakterielle Infektionen aufgrund der durch den Virus vorgeschädigten Schleimhaut. Im Vordergrund stehen Pneumokokken und Haemophilus influenzae, bei älteren Patienten auch Staphylokokken [12]. Bei Kindern kommen Mykoplasmen und Chlamydien hinzu $[8,10,13]$. Deswegen sollten ausreichend Antibiotika bevorratet sein, wobei bei den zu erwartenden Keimspektren Clarithromycin, Levofloxacin oder Moxifloxacin (alle oral) infrage kommen [12,16]. Mittel der Wahl bei Pneumokokken ist in Deutschland weiterhin Penicillin G. Bei Staphylokokken-Beteiligung wird (ggf. zusätzlich) die Gabe eines Basis- oder auch eines Zweitgenerations-Cephalosporin empfohlen.

Bevorratet werden müssen auch Schmerzmittel wie Metamizol oder Paracetamol sowie Antiemetika, da die Patienten oft erhebliche Schmerzen und Übelkeit haben können. Des Weiteren werden Sedativa (Benzodiazepine, Propofol) benötigt. Außerdem sollten Opiate vorgehalten werden, am besten Morphin, was nicht nur bei stärkeren Schmerzen von Vorteil ist, sondern auch mitunter bei der Einleitung der Beatmung zur Adaptation an die 
Beatmungsmaschine sehr hilfreich sein kann. Je nach klinischem Zustand des Patienten sind mitunter Flüssigkeitssubstitutionen erforderlich. Entsprechende Infusionslösungen sollten bevorratet werden. Eine ggf. erforderliche antiobstruktive Therapie sollte mit Dosieraerosolen durchgeführt werden. Vernebler sind aus Gründen der Infektionsausbreitung kontraindiziert.

\section{Sauerstoff}

Die Patienten sind in der Regel hypoxämisch, sichtbar an der Pulsoxymetrie. Eine Sauerstoffinsufflation ist immer ohne Nachteil, um eine arterielle Sauerstoffsättigung von $85-90 \%$ anzustreben. Inspiratorische Sauerstoffkonzentrationen von über $50 \%$ via Ventilator sollten wegen der schädigenden Wirkung vermieden werden. Mit Nasensonden ist es aber nicht möglich, diese Grenzwerte zu überschreiten. Wenn die Zimmer mit mehr Patienten belegt sind, als Sauerstoffanschlüsse zur Verfügung stehen, so kann mittels T-Stücken der Sauerstoff auf mehrere Patienten verteilt werden. Hier ist allerdings darauf zu achten, dass die Leitungen zwischen den Patienten gleich lang sind, damit am Ende etwa der gleiche Sauerstofffluss herauskommt.

\section{Beatmungsgeräte}

Die Mehrzahl der Patienten lässt sich heute auch bei der akuten respiratorischen Insuffizienz nicht-invasiv mit Masken beatmen. Ausnahmen stellen z.B. Patienten mit schwergradigem Verlauf des hypoxämischen Versagens oder okkludierende Schwellung im Pharynxbereich dar. Vorteilhaft sind kombinierte NasenMundmasken, da diese Mundleckagen verhindern. Auf jeden Fall sollten mindestens 3 Maskengrößen für Erwachsene und 1 oder 2 für Kinder zur Verfügung stehen. Hier gibt es inzwischen zahlreiche Anbieter. Natürlich muss entsprechend der Beatmungsgeräte auch mindestens die doppelte Menge an Einmalschlauchsystemen mit zwischengeschalteten Filtern bevorratet werden. Ambubeutel sollten ebenfalls in ausreichender Menge zur Verfügung stehen (etwa so viel wie Beatmungsgeräte).

Als Beatmungsgeräte reichen die Systeme für die häusliche Beatmung völlig aus. In der Regel haben sie heute eine Druckvorgabe. Ältere volumengesteuerte Geräte sind aber ebenfalls geeignet. Hierbei muss bedacht werden, dass vor allen Dingen ältere (in der Regel lautere) Geräte häufig von den Krankenkassen zurückgenommen und zentral gelagert werden. Auf diesen Pool kann man regional durchaus zugreifen, um eine entsprechende Anzahl von Beatmungsgeräten vorzuhalten. Wenn möglich sollten die Beatmungsgeräte vor der Maske und am Auslass des Gerätes einen Filter haben, damit nicht so viele Schlauchsysteme gereinigt werden müssen, wenn neue Patienten kommen. Die Filter sind in der Regel virusdicht, so dass das Innere der Beatmungsgeräte keimarm bleibt. Hinzu kommt, dass der Fluss der Geräte nicht ausreichend ist, um ein Aerosol zu erzeugen, das ggf. infektiöse Partikel tragen könnte.

\section{Praktische Durchführung der Beatmung}

Die nicht invasive Beatmung sollte in der Klinik etabliert sein, damit im Notfall entsprechendes Personal zur Verfügung steht, um weiteres Personal anzulernen. Wichtig ist, den Patienten nicht durch ungeschicktes Adaptieren der Maske zu verschrecken. Es ist deswegen vorteilhaft, dass er anfangs die Maske aufgesetzt bekommt, ohne dass ein Beatmungsschlauch oder ein Ambubeutel angeschlossen ist. Wenn sich der Patient an die
Maske gewöhnt hat, schließt man einen Ambubeutel an und versucht das Muster des Patienten mit dem Handdruck zu imitieren. Üblicherweise wird bei respiratorischer Insuffizienz dieses von dem Patienten als sehr angenehm empfunden. Dann sollte versucht werden, mit dem Beatmungsgerät das Atemmuster des Patienten zu imitieren, wobei in der Regel assistierte Beatmungsverfahren im Vordergrund stehen. Manche Patienten lassen sich aber auch gleich kontrolliert beatmen, was für eine bessere Entlastung der Atemmuskulatur vorteilhaft ist. Bei bedrohlichem Geräteengpass ist es im Einzelfall auch möglich, zwei sedierte Patienten mit kontrolliertem Beatmungsmodus über ein T-Stück zu beatmen.

Zusätzlich zur Beatmung sollte Sauerstoff appliziert werden, wenn ausreichende Mengen zur Verfügung stehen. Ansonsten sollten nur solche Personen zusätzlich Sauerstoff bekommen, die auch unter Beatmung schwerer hypoxämisch sind (Sättigungswerte unter $85 \%$ ). Die Beatmung wird überwacht durch die Pulsoxymetrie. Hier reicht z.B. ein Pulsoxymeter für $10 \mathrm{~Pa}$ tienten. Die Pflegekraft misst 1-2 Minuten die Sättigung, trägt sie ein und wechselt dann zum nächsten Patienten. Während der Spontanatmungsphase ist insbesondere die Atemfrequenz zu dokumentieren, da sie eine Verschlechterung wie auch eine Erholung am besten anzeigt.

Auf keinen Fall sollte man versuchen, hohe Sättigungswerte zu erreichen, wenn das nur mit Mühe (hoher Beatmungsdruck, hoher $\mathrm{FiO}_{2}$ ) möglich ist. Für die Gefährdung des Organismus bezüglich Hypoxie ist bei diesen Patienten allein der Sauerstoffgehalt entscheidend [9]. Dieser errechnet sich aus Sättigung $\times \mathrm{Hb} \times 1,3$. Daher können mitunter Sättigungswerte von $70-80 \%$ auch für einige Tage toleriert werden. (Kritisch hypoxisch wird der Organismus erst, wenn der Sauerstoffgehalt unter ca. $50 \%$ des Normwertes fällt, was etwa $7 \mathrm{ml} \mathrm{O} / 100 \mathrm{ml}$ Blut entspricht). Der Kohlendioxidpartialdruck bzw. der damit zusammenhängende arterielle pH-Wert ist i.d. R. unkritisch (Ausnahme: z.B. Hirnödem). Es reicht im Notfall, allein nach dem Wert bzw. dem Verlauf der Sauerstoffsättigung zu beatmen. Kritisch sind Patienten mit pulmonalen, kardialen oder anderen Vorerkrankungen aus dem Bereich der Inneren Medizin. Falls freie Betten auf der Intensivstation verfügbar sind, sollten diese Patienten dort bevorzugt aufgenommen werden. Zur Erkennung von Komplikationen bei diesen Patienten sind Blutgasanalysen i.d. R. via externes Labor empfehlenswert.

Die Maskenbeatmung hat im Gegensatz zur Intubation den Vorteil, dass der Patient sein infektiöses Sekret entfernen kann. Dazu wird die Maske kurz abgenommen, und der Patient hustet sich frei. Sollte der Hustenstoß bei Hypersekretion nicht ausreichen, das Sekret auszuwerfen, dann kann der Schleim vom Erfahrenen bronchoskopisch -während der nicht-invasiven Beatmung- unter Sicht abgesaugt werden. Falls infolge Obstruktion der oberen Atemwege oder anderen Gründen eine Maskenbeatmung nicht möglich ist, muss oral intubiert werden. Gelingt dieses nicht, ist im Notfall eine Koniotomie vorzunehmen. Einmalsets (z. B. Nutrake $^{\circledR}$ ) sind vorrätig zu halten. 


\section{Literatur}

${ }^{1}$ Antonelli M, Conti G, Moro ML, Esquinas A et al. Predictors of failure of noninvasive positive pressure ventilation in patients with acute hypoxemic respiratory failure: a multi-center study. Intensive Care Med 2001; 27: $1718-1728$

${ }^{2}$ Becker H, Schönhofer B, Buchardi H. Nicht-Invasive Beatmung. Stuttgart: Thieme, 2005

${ }^{3}$ Brochard L, Mancebo J, Elliott MW. Noninvasive ventilation for acute respiratory failure. Eur Respir J 2002; 19: 712 - 721. Review

${ }^{4}$ Brochard L. Mechanical ventilation: invasive versus noninvasive. Eur Respir J Suppl 2003; 47: 31s-37s. Review

${ }^{5}$ Carlucci A, Richard JC, Wysocki M, Lepage E, Brochard L SRLF Collaborative Group on Mechanical Ventilation. Noninvasive versus conventional mechanical ventilation. An epidemiologic survey. Am J Respir Crit Care Med 2001; 163: 874-880

${ }^{6}$ Elliott MW. Non-invasive ventilation for acute respiratory disease. $\mathrm{Br}$ Med Bull 2005; 72: 83 - 97. Print 2005

${ }^{7}$ Girou E, Schortgen F, Delclaux C et al. Association of noninvasive ventilation with nosocomial infections and survival in critically ill patients. JAMA 2000; 284: $2361-2367$

8 Juven T, Mertsola J, Waris M et al. Etiology of community-acquired pneumonia in 254 hospitalized children. Pediatr Infect Dis J 2000; 19: $293-298$
${ }^{9}$ Köhler D. Die überschätzte Hypoxämie. Pneumologie 2002; 56: 408-412. Review

${ }^{10}$ Laundy M, Ajayi-Obe E, Hawrami K et al. Influenza A communityacquired pneumonia in East London infants and young children. Pediatr Infect Dis J 2003; 22: 223-227

${ }^{11}$ Lightowler JV, Wedzicha JA, Elliott MW, Ram FS. Non-invasive positive pressure ventilation to treat respiratory failure resulting from exacerbations of chronic obstructive pulmonary disease: Cochrane systematic review and meta-analysis. BMJ 2003; 326: 185-Review

${ }^{12}$ Lorenz J, Bodmann KF, Bauer TT et al. Nosokomiale Pneumonie, Prävention, Diagnose und Therapie. Pneumologie 2003; 57: 532 - 545

${ }^{13}$ Michelow IC, Olsen K, Lozano J, Rollins NK et al. Epidemiology and clinical characteristics of community-acquired pneumonia in hospitalized children. Pediatrics 2004; 113: $701-707$

14 Oliveira EC, Lee B, Colice GL. Influenza in the intensive care unit. J Intensive Care Med 2003; 18: 80-91. Review

15 Oliveira EC, Marik PE, Colice G. Influenza pneumonia: a descriptive study. Chest 2001; 119: $1717-1723$

${ }^{16}$ Vogel F, Scholz H, al-Nawas B, Elies W, Kresken M, Lode H, Muller O, Naber KG, Petersen E, Shah P, Sorgel F, Stille W, Tauchnitz C, Trautmann M, Ullmann U, Wacha H, Wiedemann B, Paul Ehrlich Society for Chemotherapy. Rationaler Einsatz oraler Antibiotika beim Erwachsenen. Med Monatsschr Pharm 2002; 25: 193-204 